\title{
Synthetic Aperture Processing of Buried Object Scanning Sonar Data
}

\author{
Steven G. Schock \\ James Wulf \\ Gwendoline Quentin \\ Department of Ocean Engineering \\ Florida Atlantic University \\ Jason Sara \\ EdgeTech
}

\begin{abstract}
Synthetic aperture processing improves the resolution and signal to scattering noise performance of buried object scanning sonar (BOSS) imagery. Time delay focusing coherently sums acoustic data measured by a line hydrophone array located in the wings of the BOSS vehicle. Synthetic aperture processing improves the along track spatial resolution by coherently summing the data over a sequence of transmissions. SAS (synthetic aperture sonar) motion compensation is implemented by calculating the changes in projector and hydrophone positions between transmissions using IMU (inertial measurement unit) and DVL (doppler velocity log) data. The coherent summation is performed at each location in a 3D volume of focal points including the upper meter of sediments and the sediment-water interface. Sonar images are projections of the 3D data set onto orthogonal planes. A set of three cylinders with diameters of $5,7.5$ and $10 \mathrm{~cm}$, buried in sand, are used to measure the relationship between SAS aperture length and the SNR and spatial resolution of BOSS imagery. The BOSS data sets containing the cylindrical targets were collected in September 2004 as part of SAX-04 (Sediment Acoustics Experiment - 2004), located off Fort Walton Beach, Florida.
\end{abstract}

\section{I.. INTRODUCTION}

BOSS is a wideband FM sonar that generates multi-aspect imagery of buried, partially buried and proud targets using an omnidirectional projector that transmits pulses in the band of 3 to $20 \mathrm{kHz}$ and hydrophone arrays embedded into towed body wings that measure the backscattering from the seabed. Matched-filtered hydrophone data are coherently summed using time-delay focusing to form an image of the seabed. The travel time from the projector to a subsurface focal point to a hydrophone is calculated using a navigation solution based on 3 axis velocity data from a DVL and 3 axis accelerometer and rate gyro data from an IMU (inertial measurement unit). After each transmission, the focused data are stored in 3D matrix of scattering amplitudes. Maximum intensity projections of the 3D matrix data onto planes form three orthogonal views of the seabed. The maxima of a sequence of overlapping projections form a multi-aspect image projection. The sonar operator uses the three orthogonal multi-aspect image projections to view top and side views of buried targets.

In order to improve the resolution of target imagery generated by BOSS, time-delay focusing is extended to hydrophone data collected over several transmissions. Previous publications on BOSS processing discuss strip-map[1] and multi-aspect processing[2,3] based on time-delay focusing of data from a large aperture hydrophone array. In these approaches the resolution of target imagery is limited by the dimensions of the hydrophone array. With synthetic aperture processing, the along track resolution of target imagery improves with distance traveled while forming the synthetic aperture. The use of synthetic aperture processing also allows the along vehicle dimension of the array to be significantly reduced thereby reducing the hydrophone array drag and surface area and enabling the deployment of BOSS on AUVs (autononmous underwater vehicles).

\section{BACKGROUND}

\section{A. Signal processing}

Multi-aspect imagery is generated by coherently summing reflection data from all hydrophones in the physical and synthetic apertures. After each transmission, a 3D matrix of image pixels is calculated from the last $M$ pings of the synthetic aperture, starting at ping $m_{1}$ and ending at ping $m_{2}$, using the expression

$A\left(x, y, z, m_{2}\right)=\left|\frac{1}{N M} \sum_{n=1}^{N} \sum_{m=m_{2}-M+1}^{m_{2}} s_{n, m}\left(t_{T F n}\right) R_{T F m} R_{F n, m}\right|$

where $s_{n, m}\left(t_{T F n}\right)$ is the analytic signal for the nth hydrophone channel for ping $m$ where $t_{T F n}$ is the sample time associated with the path from the transmitter to the focal point $X_{F}$ to receiver $n, R_{T F m}$ is the path length from the transmitter to the focal point for ping $m$, and $R_{F n, m}$ is the path length from the focal point to the nth hydrophone for ping $m$. Fig. 1 shows a schematic of the sonar vehicle position for two transmissions and the changing position vectors of the transducer and hydrophone with respect to the focal point.

The multi-aspect image $B(x, y, z)$ is constructed by saving the maximum values of a sequence of $Q$ overlapping matrices $A\left(x, y, z, m_{2}\right)$.

$B(x, y, z)=\max \left\langle A\left(x, y, z, m_{2}-Q+1\right), \ldots, A\left(x, y, z, m_{2}\right)\right\rangle$ 


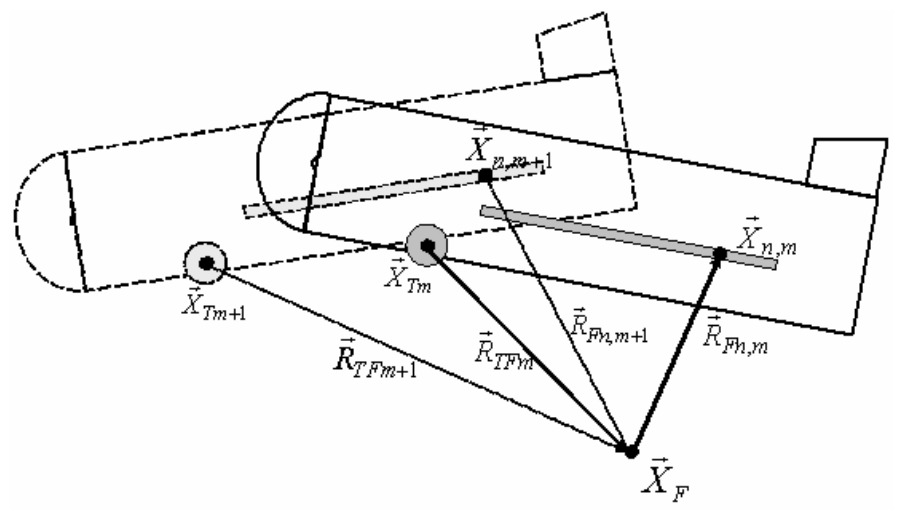

Fig. 1. Positions of projector and hydrophone $n$ with respect to a single focal point $X_{F}$ for transmissions $m$ and $m \dashv 1$.

The three orthogonal views of the seabed are given by

$$
\begin{aligned}
& P(x, y)=\max \left\langle B\left(x, y, z_{1}\right), B\left(x, y, z_{2}\right), \ldots\right\rangle \\
& P(x, z)=\max \left\langle B\left(x, y_{1}, z\right), B\left(x, y_{2}, z\right), \ldots\right\rangle \\
& P(y, z)=\max \left\langle B\left(x_{1}, y, z\right), B\left(x_{2}, y, z\right), \ldots . .\right\rangle
\end{aligned}
$$

\section{B. Image resolution}

The far field along track resolution for a synthetic aperture can be estimated from the two way aperture function of a continuous line array with rectangular weighting given by,

$b=\left|\frac{\sin (v)}{v}\right|^{2}$

where

$v=\frac{1}{2} k L \sin (\theta)$,

and $k$ is the wavenumber, $L$ is the length of the synthetic aperture and $\theta$ is the angle off the maximum response axis of the line array [4]. At the half power point of the two way aperture function, $b=1 / \sqrt{2}$ and $v=1.0$. It follows that the half power beamwidth is given by

$2 \theta_{-3 d B}=2 \sin ^{-1}\left(\frac{2}{k L}\right)$.

For $k L \gg=1$,

$2 \theta_{-3 d B}=\frac{4}{k L}=0.64 \frac{\lambda}{L}$

The resolution of a point scatterer along the maximum response axis of the line array at range $R$ is

$\delta=2 \theta_{-3 d B} R$.

As the aperture length increases, the point scatterer falls within the near field of the array. For exact synthetic aperture processing or for focusing using a discrete element array, the along track resolution approaches $\lambda / 2$ as the array length $L$ approaches range $R$.

\section{DESCRIPTION OF EXPERIMENT}

During September 2004, BOSS generated imagery of targets buried in sand at the SAX-04 site located one kilometer south of Fort Walton Beach, Florida in approximately 18 meters of water. The sonar vehicle is shown in Fig. 2. A spherical projector transmitted a $5 \mathrm{msec}$ FM pulse over the band of 3 to $20 \mathrm{kHz}$ at a rate of 10 pulses per second and illuminated the seabed across the entire 360 azimuth and for seabed incidence angles varying from normal to grazing. The reflection data was acquired with a 1.5 meter diameter circular receiving array containing 252 equally spaced hydrophones

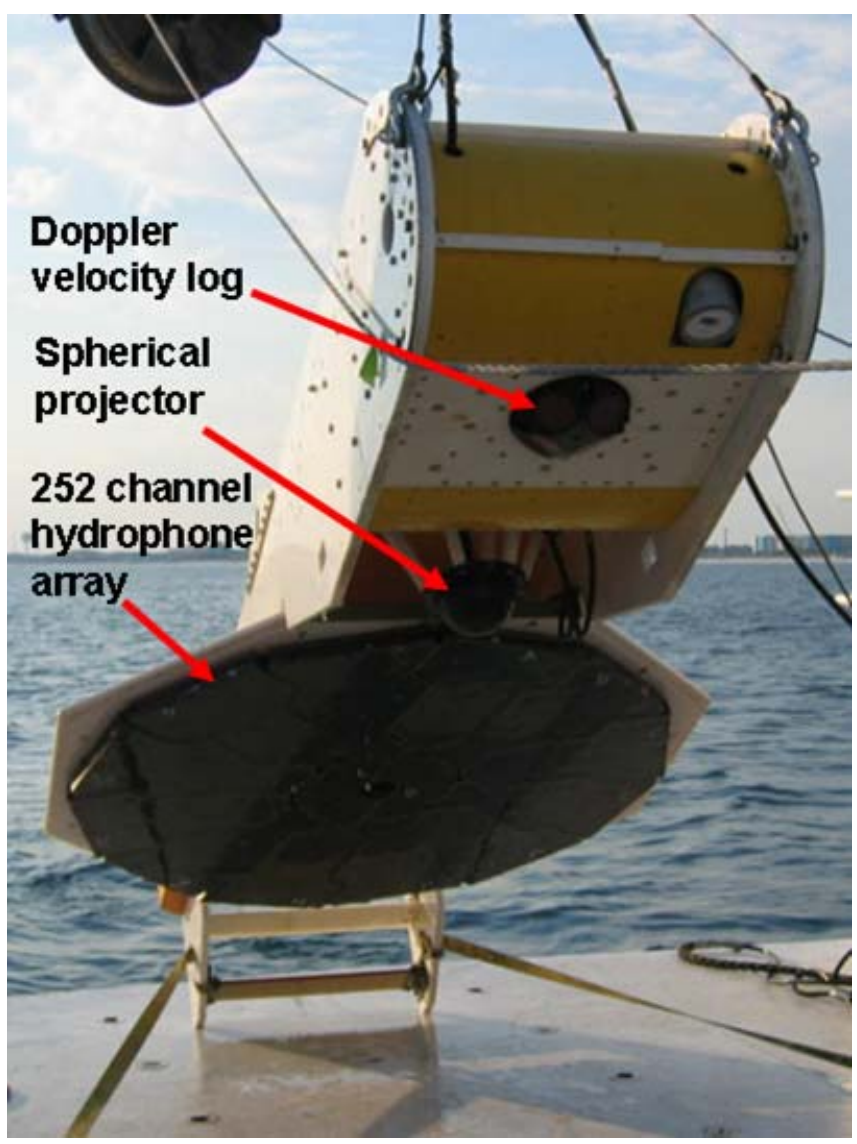

Fig. 2. Photo of BOSS sonar vehicle showing 1.5 meter diameter plane array of 252 hydrophones, spherical projector and DVL.

After cross-correlating the reflection data with the transmission pulse, a sonar processor generates three dimensional images of the seabed by coherently summing reflection data from all hydrophones in the physical and synthetic apertures (2.1). A multi-aspect image of a target is formed by saving the maximum intensities of the coherently summed data for each focal point over a sequence of transmissions (or target aspect angles) to form a multi-aspect image of a target (2.2). The multi-aspect data are projected onto three orthogonal planes for display(2.3-3.5).

A multi-aspect image of the southern side of the SAX-04 target field is shown in Figure 3 (top view only). The multi- 
aspect image shows the history of target echoes from the closest point of approach to a horizontal range of about 15 meters. During the survey, BOSS had an altitude of 4.5 meters and a velocity of 2.5 knots. The image in Fig. 3 was produced using data from only one line of 16 hydrophones oriented across the vehicle. This image as well as all subsequent images are generated using the subband of 7 to $18 \mathrm{kHz}$, the band associated with the highest target echo levels, and using a focal point interval of $10 \mathrm{~cm}$. Consequently, the interval between image pixels is $10 \mathrm{~cm}$.

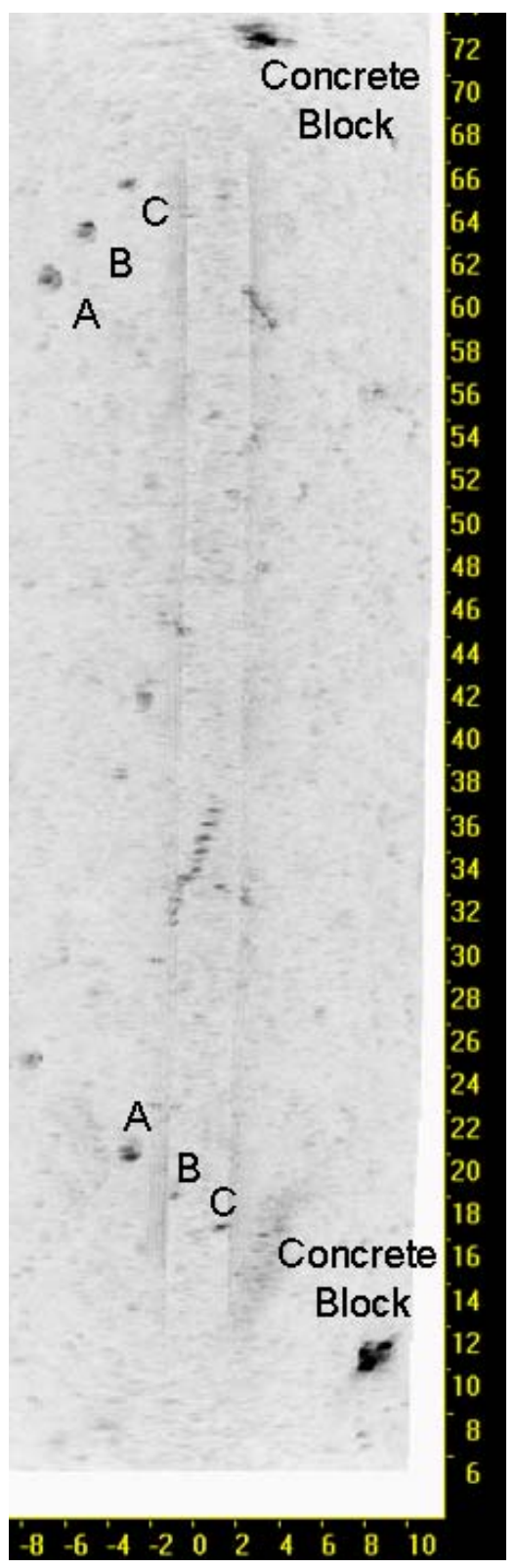

Fig. 3. Multi-aspect image of the southern edge of SAX04 target field showing concrete corner markers and concretefilled metal cylinders labeled A, B and C. The top of the image is the east side of the field. In the upper half of the image targets $\mathrm{A}, \mathrm{B}$ and $\mathrm{C}$ are buried flush with the seabed; in the lower half, the targets are buried $30 \mathrm{~cm}$.
The performance of SAS processing is evaluated using the set of three flush buried cylinders in the upper left corner of Fig. 3. Each of the flush-buried cylinders is buried horizontally (the axis of symmetry is parallel to the sediment-water interface) with the top side of the cylinder flush with the sediment-water interface. These concrete filled cylindrical metal tubes were prepared by Ray Lim, NSWC, Panama City. The lengths and diameters of the cylinders are given in Table I.

\section{TABLE I}

DIMENSIONS OF CYLINDRICAL METAL TUBES

\begin{tabular}{lll}
\hline & Length $(\mathrm{cm})$ & Diameter $(\mathrm{cm})$ \\
\hline Cylinder A & 50.5 & 10.2 \\
Cylinder B & 35.6 & 7.6 \\
Cylinder C & 30.5 & 5.1 \\
\hline
\end{tabular}

The multi-aspect image in Fig. 3 is constructed from a sequence of single-aspect images. BOSS acquired approximately 150 acoustic returns from each of the flushburied cylinders as the sonar passed by the cylinders. A subset of the 150 single-aspect images containing the three flush buried cylinders is shown in Fig. 4. The intersection of the critical angle with the seabed is indicated by the red circle in the single aspect imagery. Note that some cylinder echo data were received at ranges beyond that of the critical angle indicating possible detection by the evanscent boundary wave. There were no sand ripples observed in the sonar imagery

\section{RESULTS OF EXPERIMENT}

To establish the performance of SAS processing using motion compensation based on IMU and DVL data, BOSS data are post-processed using synthetic apertures formed using 8,15 and 30 transmissions corresponding to synthetic aperture lengths of $1.0,1.875$ and 3.75 meters, respectively. SAS performance is established by comparing the resolution and signal to noise ratios(SNR)of the flush buried cylinders in single-aspect images for the three synthetic aperture lengths.

The temporal resolution of a sonar is given by

$$
\delta_{T}=c / 2 W
$$

where $c$ is the sound speed of seawater and $W$ is the acoustic bandwidth. For a $3 \mathrm{~dB}$ bandwidth of $12 \mathrm{kHz}$, the range resolution of the target imagery should be about $6 \mathrm{~cm}$ (for a target with a flat frequency response). As seen in the last (bottom right) single-aspect image in Fig. 4, the echo off the side of the $5.1 \mathrm{~cm}$ diameter cylinder is one pixel wide indicating the temporal resolution is better or equal to $10 \mathrm{~cm}$.

The improvement in the along track resolution with increasing synthetic aperture length is measured using the along track width of the end echoes from the outer $(50.5 \mathrm{~cm}$ long) cylinder at the aspect where the cylinder is off the beam of the sonar vehicle as shown in Fig.5. The figure shows three single aspect images of the cylinder for aperture lengths of 1.0, 1.875 and 3.75 meters. The image generated using the 3.75 synthetic aperture clearly resolves the separation between the 


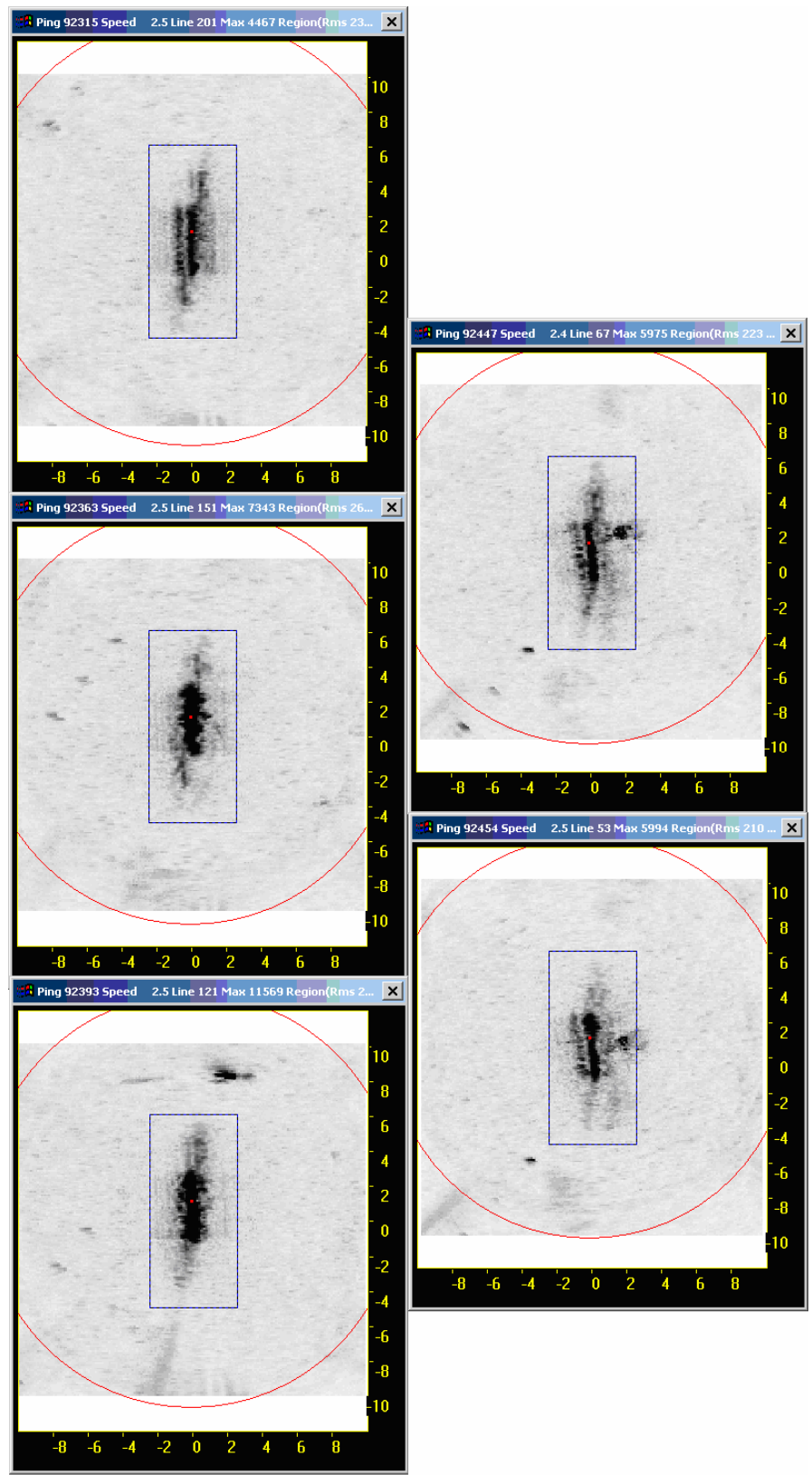

Fig. 4. Subset of 150 single-aspect images of flush-buried cylindrical targets used to construct the multi-aspect image of the targets in the upper left hand corner of Fig. 3. The red circle indicates where the critical angle intersects the seabed. The blue box indicates the region containing sediment-water interface scattering which is rejected before forming the multiaspect image. The vertical axis corresponds to the direction of the sonar track. The horizontal axis is the across track range (in meters). The sequence of five images (increasing time) is from top to bottom in the first column, then top to bottom in the second column. Each single-aspect image was formed from a 30 ping synthetic aperture.

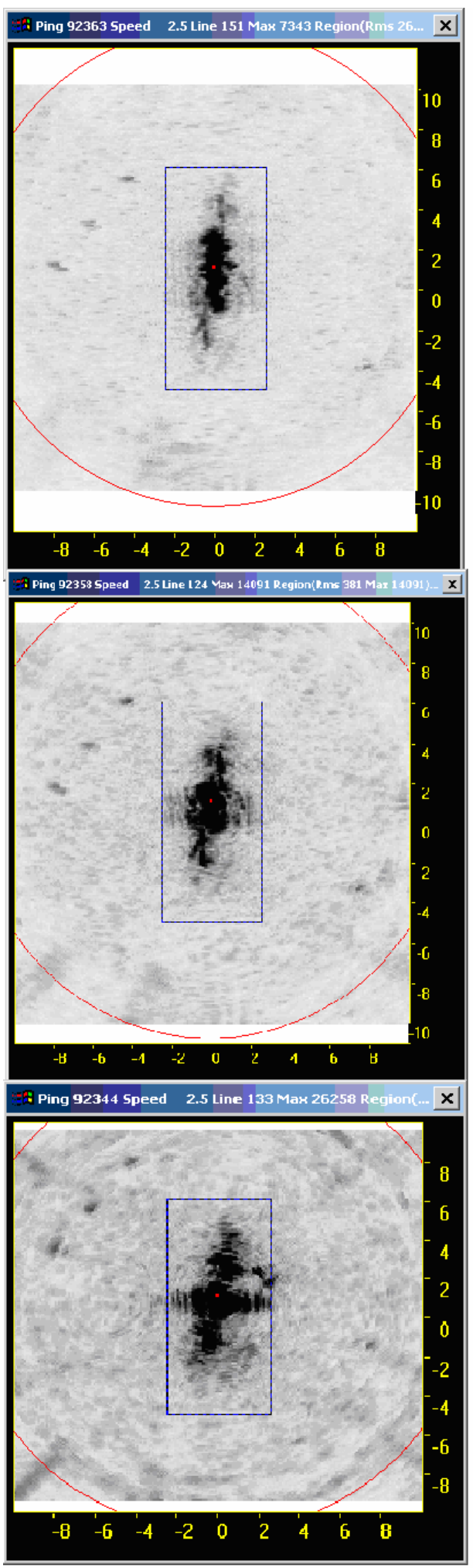

Fig. 5. Single aspect images of flush-buried cylinders for synthetic aperture lengths of 1.0 (bottom), 1.875 and 3.75 (top) meters. The horizontal and vertical scales are in meters.

end echoes of the $50.5 \mathrm{~cm}$ cylinder at a horizontal range of 8 meters (slant range of 9.2 meters). The half power width of the 
cylinder end echo is estimated to be 1, 2 and 4 pixels (10, 20 and $40 \mathrm{~cm}$ ) in the along track direction for the synthetic aperture lengths of $3.75,1,875$ and 1.0 meters. Using (2.10), a center frequency of $12.5 \mathrm{kHz}$, and a range of 9.2 meters, the expected echo widths are 19,38 and $71 \mathrm{~cm}$, respectively. The BOSS image resolution is almost a factor of two better than expected. A possible explanation for the better than expected resoluton is that the cylinder end may be preferentially backscattering energy near the upper end of the operating band $(18 \mathrm{kHz})$, thereby creating an effective imaging frequency near $18 \mathrm{kHz}$.

The single-aspect images of the cylinders in Fig. 5 also provide the SNR performance improvement obtained from synthetic aperture processing. The peak pixel amplitude of the cylinder image is compared with scattering noise measured adjacent to the cylinder for the three images (or synthetic aperture lengths). Table II summarizes the SNR measurements for the $10.2 \mathrm{~cm}$ diameter cylinder, the cylinder at the across track range of 8 meters in Fig. 5.

\section{TABLE II}

SUMMARY OF SNR MEASUREMENTS USING IMAGES OF A 10.2 CM DIAMETER CYLINDER

\begin{tabular}{lllllll}
\hline $\begin{array}{l}\text { Aperture } \\
\text { Length } \\
(\mathrm{m})\end{array}$ & $\begin{array}{l}\text { Peak } \\
\text { Echo } \\
\text { Ampl }\end{array}$ & $\begin{array}{l}\text { RMS } \\
\text { Noise }\end{array}$ & $\begin{array}{l}\text { SNR } \\
(\mathrm{dB})\end{array}$ & $\begin{array}{l}\text { SNR } \\
\text { improve- } \\
\text { ment } \\
(\mathrm{dB})\end{array}$ & $\begin{array}{l}\text { Expected } \\
\text { SNR } \\
\text { Improve- } \\
\text { ment } \\
(\mathrm{dB})\end{array}$ & $\begin{array}{l}\text { Noise } \\
\text { Improve- } \\
\text { ment } \\
(\mathrm{dB})\end{array}$ \\
\hline 1.0 & 1900 & 320 & 15.5 & 0 & 0 & \\
1.875 & 1650 & 250 & 16.4 & 0.9 & 2.7 & -2.1 \\
$3.75 \mathrm{C}$ & 1400 & 190 & 17.4 & 1.9 & 5.7 & -4.5 \\
\hline
\end{tabular}

Table II shows that the signal processing gain for scattering noise rejection is close to the expected value for a synthetic aperture while the expected SNR improvement is significantly below the expected improvement as the synthetic apeture length is increased from 1.0 to 3.75 meters. For a target with a flat complex frequency response that is independent of aspect angle, the peak echo amplitude, the second column in Table II should be independent of aperture length since the focuser output is normalized by the number of pings used to form the image (2.1). The theoretical signal processing gain of a discrete array with uncorrelated scattering noise is $10 \log (\mathrm{M})$ where $M$ is the number of elements. Table II shows that SNR should improve by $5.7 \mathrm{~dB}$ as the aperture increases from 1.0 to 3.75 meters, but it only improves by $1.9 \mathrm{~dB}$. Due to the data normalization, the scattering noise is expected to decrease by $5.7 \mathrm{~dB}$, but the noise decreases by $4.5 \mathrm{~dB}$. While the actual noise rejection is about $1 \mathrm{~dB}$ less than the expected noise rejection, the measured SNR improvement is about $3 \mathrm{~dB}$ less than the expected SNR improvement. The reduction in peak pixel amplitude for the cylinder end with increasing synthetic aperture length is likely due to changes in the ampltude and phase spectrum of the cylinder end echo causing a reduction in echo coherence as the aperture length increases. For a 3.75 meter aperture at a horizonal range of 8 meters, the bearing of the cylinder changes by 26 degrees.
The change in echo amplitude as a function of angle is plotted in Fig. 6 for the three flush-buried cylindrical targets using a 3.75 meter synthetic aperture. The image amplitude changes by almost $20 \mathrm{~dB}$ as the target aspect changes. The large variation in target echo amplitude and phase with changing target aspect, limits the processing gain of synthetic aperture processing as shown in Table II.

Note that Fig. 6. also provides the orientation of the three buried cylinders. The bearing of the maximum pixel value (processed echo level) provides the target bearing when the sonar is on the maximum response axis of the cylinder; the maxima for the three cylinder occurs when the sonar is focusing about 50 to 60 degrees behind the sonar. With the right side of the image being the south side, the axis of the buried cylinder (with a pixel maximum at -60 degrees) is aligned with 060/ 240 degrees True.
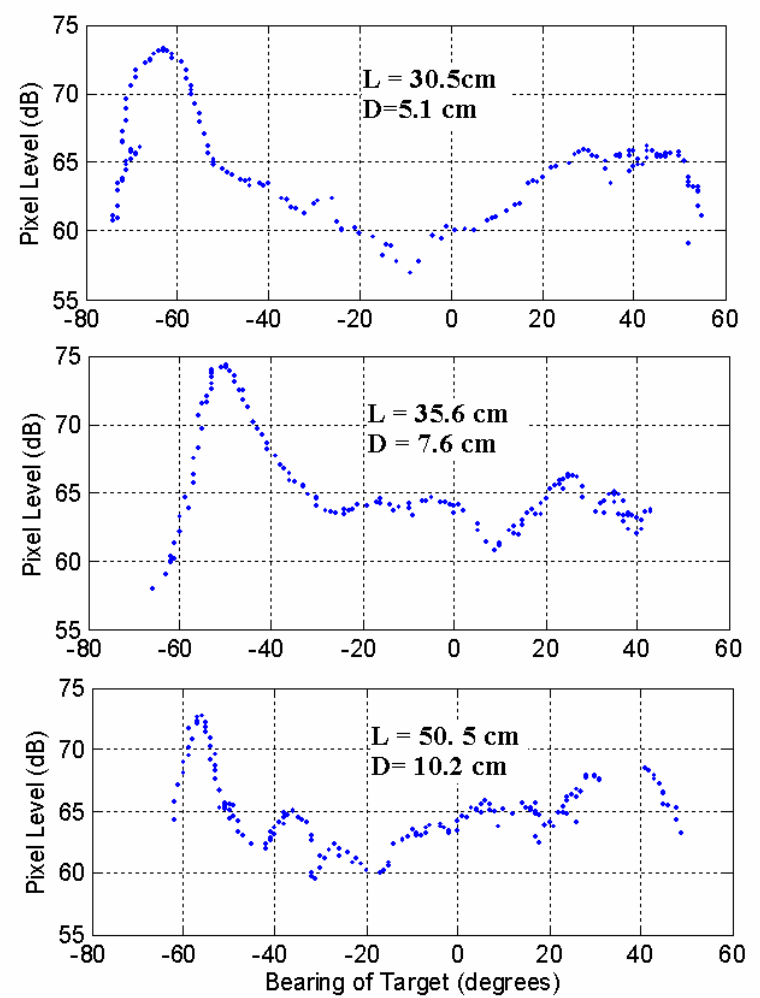

Fig. 6. Pixel levels for cylindrical targets $\mathrm{A}, \mathrm{B}$ and $\mathrm{C}$ as a function of target bearing. When the target is directly off the side of the sonar vehicle, the bearing is zero degrees. The maximum pixel value occurs when the sonar is located along the normal to the side of the cylinder. A negative angle indicates that the target is behind the sonar.

\section{CONCLUSION}

Analyses of single-aspect images generated for synthetic aperture lengths varying from 1.0 to 3.75 meters show that the along track resolution of BOSS imagery agrees with the theoretical resolution for synthetic aperture processing. The 
image analyses also show that 1) SNR improvement for synthetic aperture processing is limited by the rate at which the frequency response of the target changes with aspect angle and 2 ) the measured processing gain against scattering noise agrees with the expected processing gain $(10 \log \mathrm{M})$ for a synthetic aperture with a spatial sampling interval greater than $\lambda / 2$.

\section{ACKNOWLEDGMENT}

The authors would like to acknowledge that this research was supported by the Office of Naval Research.

\section{REFERENCES}

[1] S. Schock et al.,"Buried Object Scanning Sonar," IEEE J. of Oceanic Eng: Special Issue on Autonomous Ocean Sampling Networks, vol. 26(4), pp. 677-689, October 2001.

[2] S. Schock and J. Wulf, "Buried Object Scanning Sonar For AUVs", Proc., Oceans 2003, September 2003, San Diego.

[3] S. Schock and J. Wulf, "Sonar for Multi-Aspect Buried Mine Imaging," Proc., Oceans 2002, October 2002.

[4] J. Curlander and R. McDonough, Synthetic Aperture Radar. New York: Wiley, 1991. 